\title{
CFD modelling of two-phase discharge from a stratified region through a small side branch
}

\author{
M.K. Guyot, S.J. Ormiston, and H.M. Soliman ${ }^{\mathrm{a}}$ \\ Department of Mechanical Engineering, University of Manitoba, Winnipeg, Manitoba, Canada R3T 5V6
}

\begin{abstract}
Two-phase discharge from headers has relevance to many industrial applications, particularly in nuclear-safety analysis during postulated loss-of-coolant accidents. The main objective of this study is to assess the ability of commercial CFD codes in predicting the flow phenomena and flow parameters associated with two-phase discharge from a stratified region through a small side branch. Results were obtained for the critical heights at the onsets of gas and liquid entrainment, as well as the mass flow rate and quality during two-phase discharge. All results are in good agreement with existing experimental data. However, the computation time required to obtain these results was found to be excessive.
\end{abstract}

\section{Introduction}

Two-phase discharge from a stratified region through single or multiple branches has relevance to many industrial applications. Examples of these applications include the flow through small breaks in horizontal pipes during postulated loss-of-coolant accidents in nuclear reactors, the flow distribution in multichannel heat exchangers, and the flow distribution in two-phase headers and manifolds.

For the case of discharge from a stratified two-phase region through a single horizontal branch, Zuber [1] pointed out that two distinct flow phenomena may occur depending on the location of the gas-liquid interface relative to the branch. If the branch is located above the horizontal interface, liquid can be entrained in the predominantly gas flow, and this phenomenon is referred to as "liquid entrainment". On the other hand, if the branch is located below the horizontal interface, gas can be pulled through the branch (gas entrainment phenomenon). When the position of the interface relative to the branch is somewhere between the positions for the onset of liquid entrainment (OLE) and the onset of gas entrainment (OGE), a two-phase mixture flows into the branch.

Several experimental (e.g., Micaelli and Memponteil [2]; Yonomoto and Tasaka [3]; Hassan et al. [4]) and theoretical (e.g., Craya [5]; Soliman and Sims [6]; Saleh et al. [7]) investigations were reported for the determination of the critical heights of the interface at the OLE and OGE. These studies produced the following formulation for both critical heights:

$$
h / d=C_{1} \mathrm{Fr}^{C_{2}},
$$

where, $h$ is the critical height for OLE or OGE (measured from the centerline of the branch), $d$ is the branch diameter, Fr is Froude number, and $C_{1}$ and $C_{2}$ are coefficients whose values depend on the type of the phenomenon. The theoretical studies were in general good agreement with the experimental data.

For the condition where the interface is located between the two critical heights, experimental data were obtained for the mass flow rate and quality of the twophase mixture exiting through the branch (Yonomoto and Tasaka [3]; Hassan et al. [4]). Empirical correlations were obtained from these data (e.g., [4]); however, there have been no theoretical studies, analytical or numerical, for predicting these data. The objective of the present study is to develop numerical models for predicting the critical heights at the OLE and OGE, as well as the mass flow rate and quality in the two-phase region and to validate the numerical results in terms of magnitude and trend against existing experimental data.

\section{Numerical model}

The geometry under consideration, shown in Fig. 1, is a large rectangular tank $(246 \times 125 \times 720 \mathrm{~mm})$ with a small side branch of square cross-section $(6 \times 6 \mathrm{~mm})$ and length $120 \mathrm{~mm}$. Air and water at room temperature are contained in the tank with a flat interface. The height of the interface, $h$, is measured from the centerline of the branch ( $h$ is positive if the interface is located above the branch centerline and negative if the interface is located beneath the branch centerline). The boundary conditions at the top and bottom of the tank, and at the branch outlet depended on the type of phenomena under consideration.

\footnotetext{
a Corresponding author: hassan.soliman@ad.umanitoba.ca
} 
For the tests designed to determine the critical height for OLE, a zero pressure was imposed at the branch outlet, a steady pressure $P_{\mathrm{o}}$ (whose value depended on the desired Fr) was imposed at the top surface, and water was introduced slowly through the bottom of the tank raising the interface level at a rate of $5 \mathrm{~mm} / \mathrm{min}$. Starting with an interface sufficiently lower than the branch, a transient solution was advanced until liquid started to flow into the branch and the value of $h$ at this instant was set as $h_{\text {OLE }}$. Several tests of this type were conducted using various values of $P_{\mathrm{o}}$ (each $P_{\mathrm{o}}$ corresponds to a different Fr). For the tests designed to determine the critical height for OGE, a zero pressure was imposed at the branch outlet, a steady pressure $P_{\mathrm{o}}$ (whose value depended on the desired Fr) was imposed at the top surface, and water was introduced through the bottom of the tank at a rate equal to $99 \%$ of the rate exiting through the branch, thus slowly lowering the interface level. Starting with an interface sufficiently higher than the branch, a transient solution was advanced until air started to flow into the branch and the value of $h$ at this instant was set as $h_{\mathrm{OGE}}$. Several tests of this type were conducted using various values of $P_{0}$. For tests involving two-phase flow through the branch, a liquid height $(h)$ was established inside the tank such that $h_{\mathrm{OLE}}<h<h_{\mathrm{OGE}}$. A steady pressure $P_{\mathrm{o}}$ was imposed at the top surface and a zero pressure was imposed at the branch outlet. Water was introduced at the bottom of the tank at a rate that was successively adjusted to match the water flow rate exiting the branch. Iteration continued until all values of $h, P_{0}$, and mass flow rates of air and water entering and exiting the flow domain became steady with time. For each fixed value of $P_{\mathrm{o}}$, a number of tests were conducted using different values of $h$.

The flow domain was divided into a number of control volumes using a structured, hexahedral mesh. The mesh was refined towards the interface, the branch inlet, and in the branch itself. ANSYS CFX 14.5 was used in solving the discretized form of the governing mass, momentum, and volume- conservation equations. The $k-\varepsilon$ model was used to model the turbulence in both phases and an Eulerian-Eulerian approach was selected treating the flow as an inhomogeneous mixture with each fluid having its own velocity and turbulence fields with interaction between the phases via interfacial transfer terms. A parameter that was found to have a significant effect on the onset results is the drag coefficients used in calculating the interphase drag force. This parameter was set at 0.05 throughout this study.

Mesh independence tests were conducted in order to assess the accuracy of the numerical results. A sample of these results, corresponding to $h=-14.98 \mathrm{~mm}$ and $P_{\mathrm{o}}=$ $120 \mathrm{kPa}$ are shown in Table 1 . These results show that the deviation between the medium and fine meshes is $1.43 \%$ in $\dot{m}_{\mathrm{L}, \text { out }}$ and $0.11 \%$ in $\dot{m}_{\mathrm{G} \text {,out }}$, where $\dot{m}_{\mathrm{L} \text { out }}$ and $\dot{m}_{\mathrm{G} \text {,out }}$ are the liquid and gas mass flow rates at the outlet of the branch. Based on these results, it was decided to use mesh sizes of 1.3 to 1.8 million nodes in generating the results. In each test case, convergence was assumed when the global mass and momentum imbalances were less than $0.01 \%$ and the velocity and volume fraction at a selection of points located throughout the branch had levelled off to a constant value.

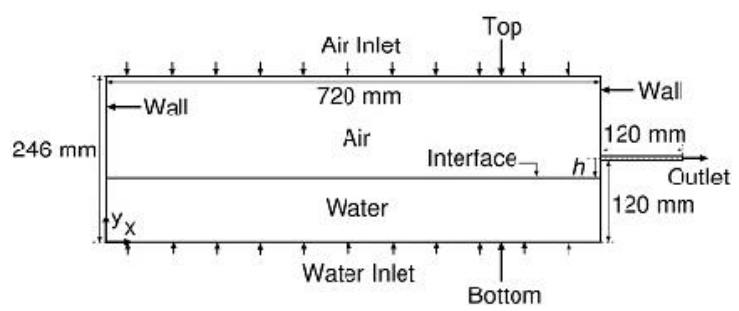

Figure 1. Geometry and coordinate system.

Table 1. Mesh-independence results

\begin{tabular}{|l|c|c|}
\hline & $\dot{m}_{\mathrm{L}, \text { out }}, \mathrm{kg} / \mathrm{min}$ & $\dot{m}_{\mathrm{G}, \text { out }}, \mathrm{kg} / \mathrm{min}$ \\
\hline $\begin{array}{l}\text { Course } \\
(0.86 \text { M nodes })\end{array}$ & 0.1960 & 0.3612 \\
\hline $\begin{array}{l}\text { Medium } \\
(1.2 \text { M nodes })\end{array}$ & 0.1770 & 0.3549 \\
\hline $\begin{array}{l}\text { Fine } \\
(3.3 \text { M nodes })\end{array}$ & 0.1745 & 0.3545 \\
\hline
\end{tabular}

\section{Results and discussion}

\subsection{Flow parameters}

For a fixed value of $P_{\mathrm{o}}$, Fig. 2 shows the dependence of the mass flow rate from the branch on the location of the interface, $h$. At high interface levels above the branch, the discharge from the branch will be in the form of singlephase liquid. Lowering the interface level, a critical height is reached where the onset of gas entrainment (OGE) occurs at the branch ( $h=h_{\mathrm{OGE}}$ and $\left.\dot{m}=\dot{m}_{\mathrm{L}, \mathrm{OGE}}\right)$. A further lowering of the interface results in a two-phase discharge through the branch at a rate $\dot{m}_{\mathrm{TP}}$. As $h$ decreases, $\dot{m}_{\mathrm{TP}}$ decreases while the quality of the twophase mixture, $x$, increases. Finally, with further lowering of the interface, another critical condition is reached (onset of liquid entrainment) where liquid stops flowing into the branch $\left(h=h_{\mathrm{OLE}}\right.$ and $\left.\dot{m}=\dot{m}_{\mathrm{G}, \mathrm{OLE}}\right)$. Beyond this point, only single-phase gas flows into the branch.

\subsection{Onset of liquid entrainment}

The onset of liquid entrainment height, $h_{\mathrm{OLE}}$, was determined at four different values of $P_{0}$, each corresponding to a different combination of $\dot{m}_{\mathrm{G}, \mathrm{OLE}}$ and gas Froude numbers, $\mathrm{Fr}_{\mathrm{G}, \mathrm{OLE}}$, where,

$$
\mathrm{Fr}_{\mathrm{G}, \mathrm{OLE}}=\frac{\dot{m}_{\mathrm{G}, \mathrm{OLE}}}{A_{\mathrm{c}} \sqrt{g d \rho_{\mathrm{G}}\left(\rho_{\mathrm{L}}-\rho_{\mathrm{G}}\right)}},
$$




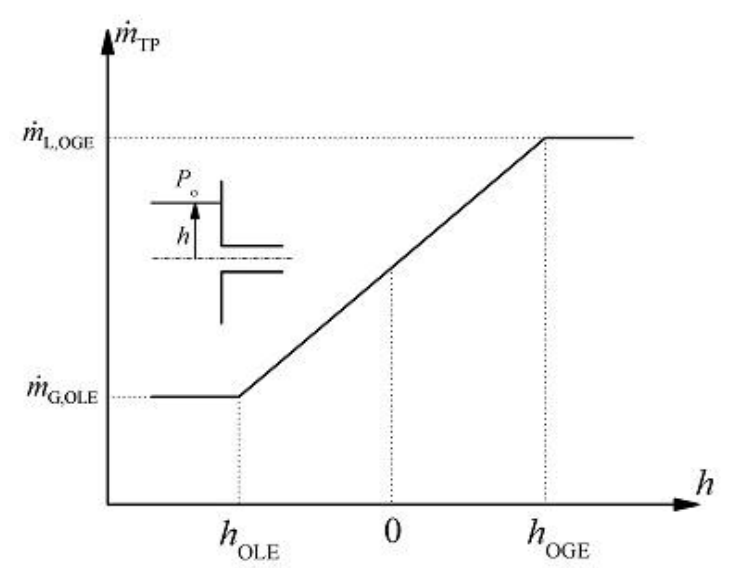

Figure 2. Variation of $\dot{m}_{\mathrm{TP}}$ with $h$.

$A_{\mathrm{c}}$ is the branch cross-sectional area, $g$ is the gravitational acceleration, $d$ is the hydraulic diameter of the branch, $\rho_{\mathrm{G}}$ is the air density, and $\rho_{\mathrm{L}}$ is the water density. Results were obtained at $\mathrm{Fr}_{\mathrm{G}, \mathrm{OLE}}=3.92,7.74,23.56$, and 32.83. Figure 3 (a) and (b) shows air volume fraction contours from the transient results for $\mathrm{Fr}_{\mathrm{G}, \mathrm{OLE}}=7.74$. The red region in these figures represents $100 \%$ air and the blue region represents $100 \%$ water. From these results it can be seen that in Fig. 3(a) when $h=-10.75 \mathrm{~mm}$, the interface is located far beneath the branch inlet and by raising the interface slightly to $h=-10.70 \mathrm{~mm}$, Fig. 3(b) shows that the water beneath the branch inlet climbs the wall and small amounts of water exits through the branch (OLE). It is interesting to note that this phenomenon occurs very quickly (within a $0.05-\mathrm{mm}$ rise of the interface), consistent with previous experimental observations [1-4].

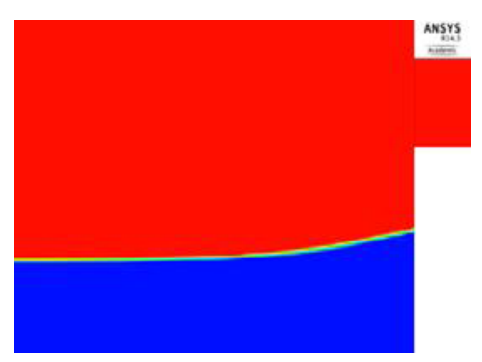

(a) $h=-10.75 \mathrm{~mm}$

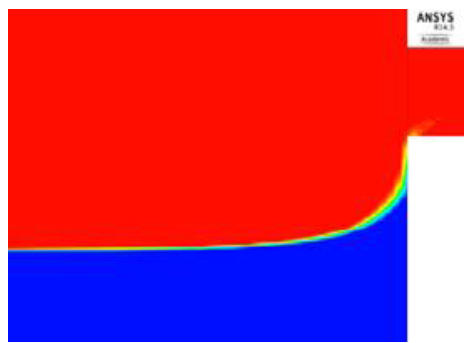

(b) $h=-10.70 \mathrm{~mm}$

Figure 3. Air volume fraction contours for $\mathrm{Fr}_{\mathrm{G}, \mathrm{OLE}}=7.74$
The results of $\left|h_{\mathrm{OLE}}\right| / d$ are plotted vs. $\mathrm{Fr}_{\mathrm{G}, \mathrm{OLE}}$ in Fig. 4 , along with the correlations developed by Yonomoto and Tasaka [3], Hassan et al. [4], and Craya [5]. The following correlation was obtained to fit the present numerical data:

$$
\left|h_{\mathrm{OLE}}\right| / d=0.808 \mathrm{Fr}_{\mathrm{G}, \mathrm{OLE}}^{0.39}
$$

The discrepancy seen in Fig. 4 between the present correlation and the previous correlations is small. In view of the difference in the branch geometry (square in this study and circular in previous studies), the result seen in Fig. 4 is satisfactory.

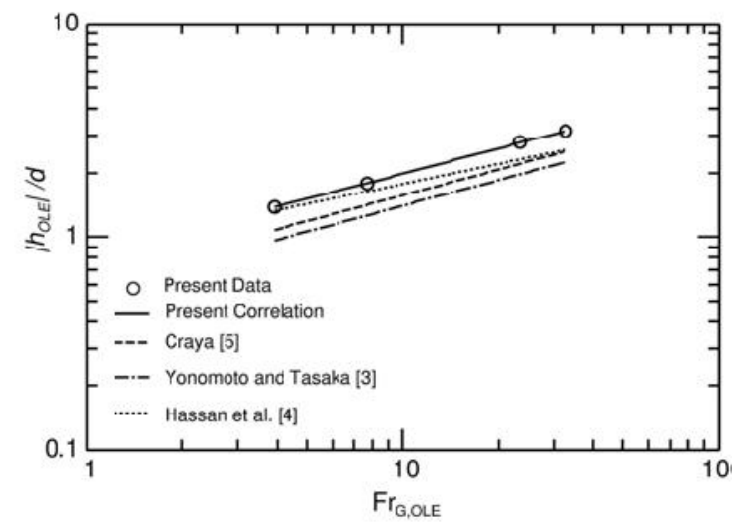

Figure 4. Interface height at the onset of liquid entrainment

\subsection{Onset of gas entrainment}

Results were obtained for the onset of gas entrainment height $h_{\mathrm{OGE}}$ for four different liquid Froude numbers: $\mathrm{Fr}_{\mathrm{L}, \mathrm{OGE}}=14.02,24.34,31.48$ and 45.75 , where $\mathrm{Fr}_{\mathrm{L}, \mathrm{OGE}}$ is given by

$$
\mathrm{Fr}_{\mathrm{L}, \mathrm{OGE}}=\frac{\dot{m}_{\mathrm{L}, \mathrm{OGE}}}{A_{\mathrm{c}} \sqrt{g d \rho_{\mathrm{L}}\left(\rho_{\mathrm{L}}-\rho_{\mathrm{G}}\right)}} .
$$

Figure 5 (a) to (d) shows the transient results of the air volume fraction contours for $\mathrm{Fr}_{\mathrm{L}, \mathrm{OGE}}=31.48$. The instance at which the onset of gas entrainment occurred was determined by monitoring the air volume fraction at the top of the branch inlet along the symmetry plane in the transient solution. At the onset, the air volume fraction at this location changed from 0 to 1 . The phenomenon shown in Fig. 5 occurred almost instantaneously while the interface level dropped by less than $0.05 \mathrm{~mm}$. As the interface drops close to the onset height, the interface begins to dip near the wall above the branch inlet and a gas cone forms extending from the interface to the branch inlet without contacting the wall. A very slight further drop in the interface height causes the gas cone to break and the air to flow freely along the wall above the branch as it enters the branch inlet. These observations are again consistent with the observations from previous experiments. 


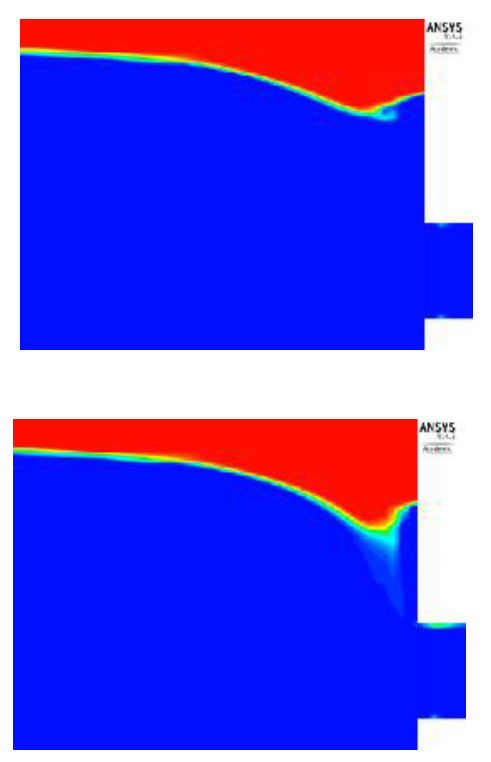

(a) $h=14.55 \mathrm{~mm}$

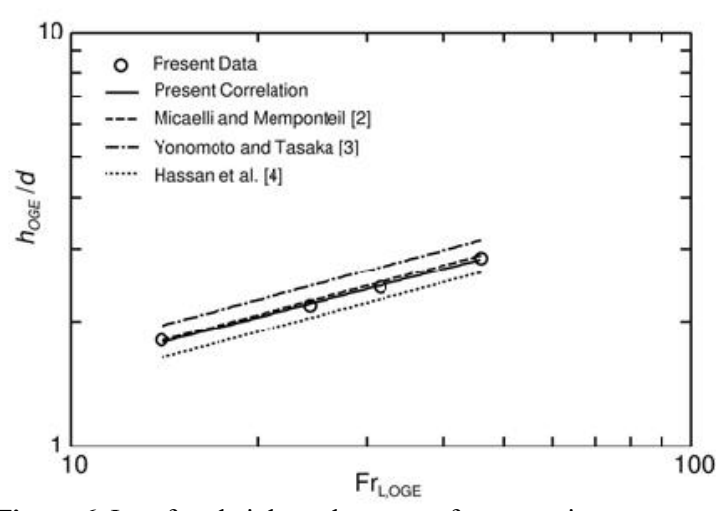

Figure 6. Interface height at the onset of gas entrainment.

(b) $h=14.55 \mathrm{~mm}$

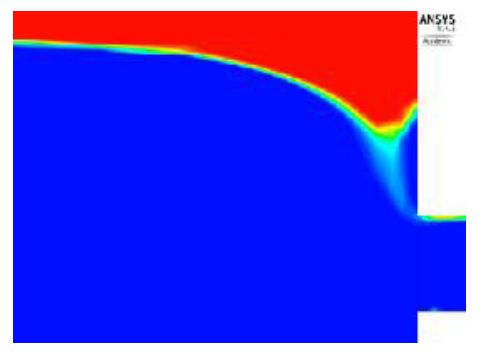

(c) $h=14.55 \mathrm{~mm}$

\subsection{Two-phase flow}

When the interface is located above $h_{\mathrm{OLE}}$ and below $h_{\mathrm{OGE}}$, both air and water flow through the branch. Under these conditions, results for the total mass flow rate, $\dot{m}_{\mathrm{TP}}$, and mixture quality, $x$, at the branch outlet were obtained for various interface heights at two tank pressures: $P_{\mathrm{o}}=35$ $\mathrm{kPa}$ and $120 \mathrm{kPa}$. Figure 7 shows $\dot{m}_{\mathrm{TP}}$ vs. $h$ for the two values of $P_{\mathrm{o}}$. For each $P_{\mathrm{o}}$, data are presented from $h=$ $h_{\mathrm{OLE}}$ to $h=h_{\mathrm{OGE}}$. From this figure it can be seen that as $P_{\mathrm{o}}$ increases, $\dot{m}_{\mathrm{TP}}$ increases for the same interface height. As well, $h_{\mathrm{OGE}}$ and $\left|h_{\mathrm{OLE}}\right|$ increase as $P_{\mathrm{o}}$ increases.

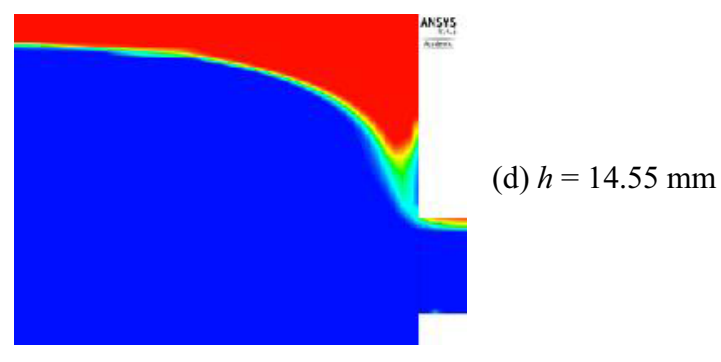

Figure 5. Air volume fraction contours for $\mathrm{Fr}_{\mathrm{L}, \mathrm{OGE}}=31.48$.

Figure 6 shows the numerical results of $h_{\mathrm{OGE}} / d$ vs. $\mathrm{Fr}_{\mathrm{L}, \mathrm{OGE}}$ along with the correlations of Micaelli and Memponteil [2], Yonomoto and Tasaka [3] and Hassan et al. [4]. The numerical data were fit by the following correlation, which is shown in Fig. 6 as a solid line:

$$
h_{\mathrm{OGE}} / d=0.636 \mathrm{Fr}_{\mathrm{L}, \mathrm{OGE}}^{0.39} \text {. }
$$

From Fig. 6 it can be seen that the present results fall within the predictions of existing empirical correlations.

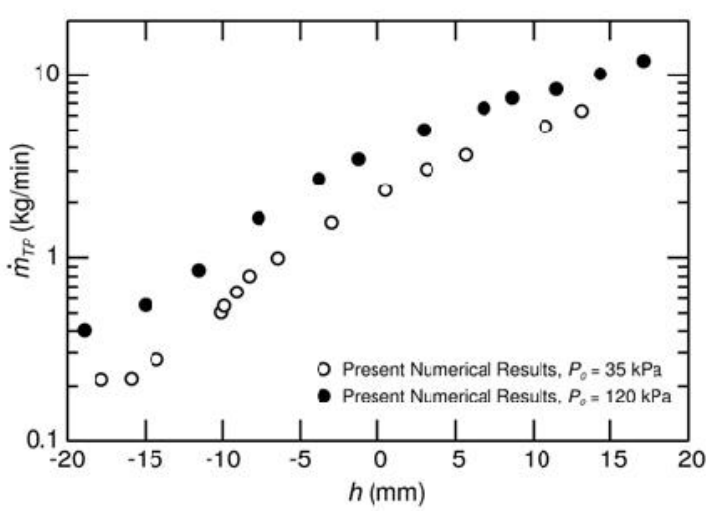

Figure 7. $\dot{m}_{\mathrm{TP}}$ vs. $h$ for $P_{0}=35$ and $120 \mathrm{kPa}$.

Hassan et al. [4] found that with their experimental data for discharge flow through a single horizontal branch, the effect of $P_{\mathrm{o}}$ was absorbed by plotting their data in terms of the dimensionless parameters $\mathrm{M}$ and $\mathrm{H}$, where

$$
\mathrm{H}=\left(h-h_{\mathrm{OLE}}\right) /\left(h_{\mathrm{OGE}}-h_{\mathrm{OLE}}\right),
$$




$$
\mathrm{M}=\left(\dot{m}_{\mathrm{TP}}-\dot{m}_{\mathrm{G}, \mathrm{OLE}}\right) /\left(\dot{m}_{\mathrm{L}, \mathrm{OGE}}-\dot{m}_{\mathrm{G}, \mathrm{OLE}}\right) .
$$

Based on their experimental data, they developed empirical correlations for $\mathrm{M}$ as a function of $\mathrm{H}$ and $x$ as a function of $\mathrm{H}$. The present numerical results for $P_{\mathrm{o}}=35$ $\mathrm{kPa}$ and $120 \mathrm{kPa}$ were plotted in Fig. 8 in terms of M vs. $\mathrm{H}$ and in Fig. 9 in terms of $x$ vs. H. The correlations of Hassan et al. [4] are shown in these figures as solid lines. From these figures it can be seen that the present numerical data also collapse when plotted using the dimensionless variables defined by Eqs. (6) and (7). In addition, the present numerical results show very good agreement with Hassan et al.'s correlations.

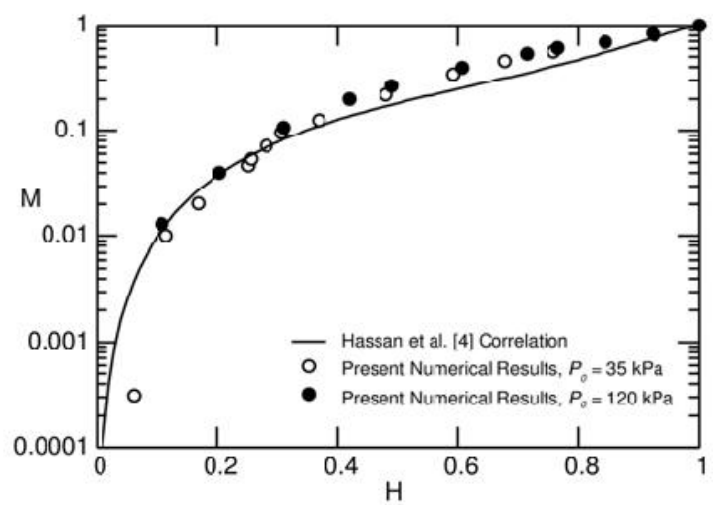

Figure 8. $\mathrm{M}$ vs. $\mathrm{H}$ for $P_{0}=35$ and $120 \mathrm{kPa}$.

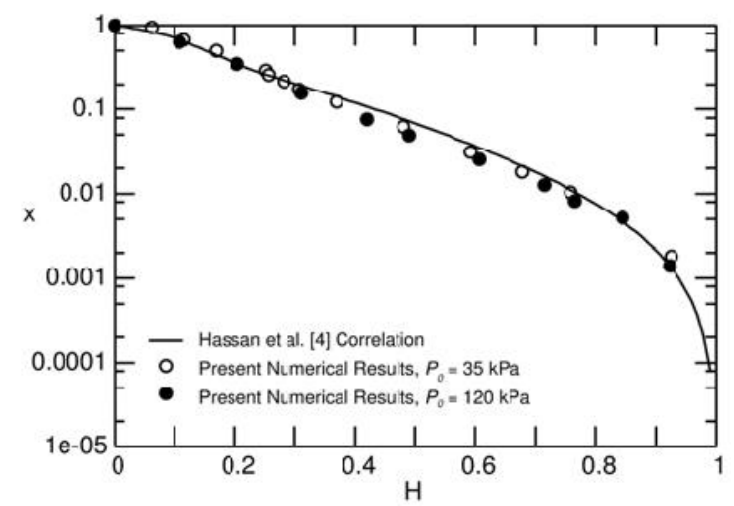

Figure 9. $x$ vs. $\mathrm{H}$ for $P_{0}=35$ and $120 \mathrm{kPa}$.

\section{Conclusion}

A numerical study was conducted on the discharge from a stratified two-phase region through a small side branch using a commercial CFD code (ANSYS CFX 14.5). Results were obtained for the flow phenomena of OLE and OGE, the critical heights $h_{\mathrm{OLE}}$ and $h_{\mathrm{OGE}}$, the mass flow rate $\dot{m}_{\mathrm{TP}}$, and quality $x$ of the two-phase discharge. These results agreed well in magnitude and trend with existing experimental data. Thus, it can be concluded that ANSYS CFX 14.5 is capable of predicting the flow phenomena for the geometry under consideration. This study is only a first step and it should be followed by more work involving more elaborate geometries.

One drawback is that, even for the present simple geometry, the computation time required for obtaining accurate converged results was found to be excessive (in the order of weeks for one data point using 8 computer cores). In order to avoid divergence, it was found that very small time steps $\left(10^{-4}\right.$ to $10^{-3}$ seconds) and large number of nodes (1.3 to 1.8 million nodes) were required. This can pose a problem in industry when dealing with more sophisticated geometries.

\section{Acknowledgement}

The financial assistance provided by the Natural Sciences and Engineering Research Council of Canada (NSERC) is gratefully acknowledged.

\section{References}

1. N. Zuber, Problems in Modeling of Small Break LOCA, Nuclear Regulatory Commission Report NUREG-0724, 1980.

2. J.C. Micaelli and A. Memponteil, Two-Phase Flow Behavior in a Tee-Junction - the CATHARE Model, In: Proceedings of the $4^{\text {th }}$ International Topical Meeting on Nuclear Reactor ThermalHydraulics, Karlsruhe, F.R.G., vol. 2, pp. 1024$1030,1989$.

3. T. Yonomoto and K. Tasaka, Liquid and Gas Entrainment to a Small Break Hole from a Stratified Two-Phase Region, International Journal of Multiphase Flow 17, 745-765 (1991).

4. I.G. Hassan, H.M. Soliman, G.E. Sims, and J.E. Kowalski, Two-Phase Flow from a Stratified Region Through a Small Side Branch, Journal of Fluids Engineering 120, 605-612 (1998).

5. A. Craya, Theoretical Research on the Flow of NonHomogeneous Fluids, La Houille Blanche 4, 44-55 (1949).

6. H.M. Soliman and G.E. Sims, Theoretical Analysis of the Onset of Liquid Entrainment for Orifices of Finite Diameter, International Journal of Multiphase Flow 18, 229-235 (1992)

7. W. Saleh, R.C. Bowden, I.G. Hassan, and L. Kadem, A Hybrid Model to Predict the Onset of Gas Entrainment with Surface Tension Effects, Journal of Fluids Engineering 131, 1-10 (2009). 